\title{
PARTITIONS OF LARGE MULTIPARTITES WITH CONGRUENCE CONDITIONS. I
}

\author{
BY
}

\author{
M. M, ROBERTSON AND D. SPENCER
}

\begin{abstract}
Let $p\left(n_{1}, \ldots, n_{j}: A_{1}, \ldots, A_{j}\right)$ be the number of partitions of $\left(n_{1}, \ldots, n_{j}\right)$ where, for $1<l<j$, the lth component of each part belongs to the set $A_{l}=\bigcup_{h(l)=1}^{q(l)}\left\{a_{l h(l)}+M \nu: \nu=0,1,2, \ldots\right\}$ and $M, q(l)$ and the $a_{l n(l)}$ are positive integers such that $0<a_{l 1}<\cdots<a_{l q(l)}$ $<M$. Asymptotic expansions for $p\left(n_{1}, \ldots, n_{j}: A_{1}, \ldots, A_{j}\right)$ are derived, when the $n_{l} \rightarrow \infty$ subject to the restriction that $n_{1} \cdots n_{j}<n j+1-\epsilon$ for all $l$, where $\epsilon$ is any fixed positive number. The case $M=1$ and arbitrary $j$ was investigated by Robertson [10] while several authors between 1940 and 1960 investigated the case $j=1$ for different values of $M$.
\end{abstract}

1. Introduction. Many authors have evaluated the number of different partitions of a multipartite number. A multipartite number of order $j$ is a $j$-dimensional vector, the components of which are positive integers, and a partition of $\left(n_{1}, \ldots, n_{j}\right)$ is a solution of the vector equation

$$
\sum_{k}\left(n_{1 k}, \ldots, n_{j k}\right)=\left(n_{1}, \ldots, n_{j}\right)
$$

in multipartites. Two partitions which differ only in the order of the multipartites on the left-hand side of (1.1) are regarded as identical.

In [10] Robertson, extending results of Wright [13], obtained asymptotic expansions for the number of different partitions of $\left(n_{1}, \ldots, n_{j}\right)$ when $n_{1} \ldots$ $n_{j}<n_{i}^{i+1-\epsilon_{1}}$ for all $l$ where $\epsilon_{1}$ is any fixed positive number less than 1 . In this article, we extend these results and obtain, subject to the same conditions on the $n_{l}$, asymptotic expansions for $p\left(n_{1}, \ldots, n_{j}: A_{1}, \ldots, A_{j}\right)$ the number of different partitions of $\left(n_{1}, \ldots, n_{j}\right)$ where, for $1 \leqslant l \leqslant j$, the lth component of each part belongs to the set

$$
A_{l}=\bigcup_{h(l)=1}^{q(l)}\left\{a_{l n(l)}+M v: v=0,1,2, \ldots\right\}
$$

Received by the editors January 29, 1975.

AMS (MOS) subject classifications (1970). Primary 10J 20, 10A45; Secondary 10A10. Key words and phrases. Partition, asymptotic, multipartite, congruence. 
and $M, q(l)$ and the $a_{l h(l)}$ are positive integers such that, for every $l, 0<a_{l 1}<$ $\cdots<a_{l q(l)} \leqslant M$.

The residue classes in which the different components of each part must lie may, in general, be expressed in terms of different moduli, and also the permissible residue classes for any particular component may be expressed in terms of different moduli. These generalizations are, however, only superficial, as all the residue classes may be expressed in terms of residue classes modulo $M$, the least common multiple of the different moduli.

In [13] there is a fairly comprehensive list of papers concerned with the asymptotic evaluation of the number of partitions of multipartites. The only investigation, of which the authors are aware, concerning multipartites subject to congruence conditions is that of Passi [8]. This paper generalizes the partition problem to lattices but obtains an asymptotic evaluation not of the number of partitions but only of its logarithm.

The case $j=1$ with different congruence conditions has been investigated by several authors. The Hardy-Ramanujan circle method as modified by Rademacher [9] has been employed to evaluate $p\left(n_{1}: A_{1}\right)$, where $q(1)=2, a_{12}=$ $M-a_{11}$, as a convergent series. The case $M=6$ was obtained by Niven [7], $M=5$ by Lehner [5] and Livingood [6] solved the case where $M$ is any prime >3. Later Iseki [3] evaluated $p\left(n_{1}: A_{1}\right)$ when $M$ is composite $>3$ and $\left(a_{11}, M\right)$ $=1$. Hagis [2] evaluated $p\left(n_{1}: A_{1}\right)$ for all odd primes $M$, where $q(1)=2 r$ and $a_{1 h}+a_{1 k}=M$ whenever $h+k=2 r$.

In all the cases mentioned in the preceding paragraph, $A_{1}$ is symmetrical in the sense that $a_{1} \in A_{1}$ implies that $M-a_{1} \in A_{1}$, and this ensures that the generating function of the $p\left(n_{1}: A_{1}\right)$ is a modular form. Rademacher's method then leads to a convergent series representation of $p\left(n_{1}: A_{1}\right)$. Grosswald [1] considered the case where $M$ is any odd prime and $A_{1}$ is an arbitrary asymmetrical set. Then the above method cannot be applied and only asymptotic results are obtained.

2. Notation and definitions. Throughout this article, $a, d, \Delta, h, k, K, l, m$, $M, n, N, \nu, q, r, \rho, s$ represent nonnegative integers and $j$ is used for an integer greater than unity. $C$ is a positive number, not necessarily the same at each oc. currence, which may depend upon any $j, M, \epsilon_{l}$ but not upon any $n_{l}, x_{l}, y_{l}, \theta_{l}$, $\xi_{l}, z_{l}$. The numbers $\epsilon_{l}$ are positive and to be thought of as small. The symbols $\sim, O\left(\right.$ ) always refer to the passage of the $n_{l}$ to infinity. The symbol $O($ ) sometimes refers to the passage of the $n_{l}$ to infinity and otherwise is obvious from the context. The total differential operator $d / d t$ is always denoted by $D$ and never by a prime. $\gamma, \zeta()$ represent respectively the Euler constant and the Riemann zeta function.

We write 
PARTITIONS OF LARGE MULTIPARTITES WITH CONGRUENCE CONDITIONS 301

$$
\begin{aligned}
& f\left(x_{1}, \ldots, x_{j}\right)=f\left(x_{1}, \ldots, x_{j}: A_{1}, \ldots, A_{j}\right) \\
& =\prod_{\nu_{1}=0}^{\infty} \cdots \prod_{\nu_{j}=0}^{\infty} \prod_{h(1)=1}^{q(1)} \cdots \prod_{h(j)=1}^{q(j)}\left(1-\exp \left(-\sum_{l=1}^{j}\left(M \nu_{l}+a_{l h(l)}\right) x_{l}\right)\right)^{-1}
\end{aligned}
$$

where $\operatorname{Re}\left(x_{l}\right)>0$ for $1 \leqslant l \leqslant j$. Writing $p(0, \ldots, 0)=1$ and $p\left(n_{1}, \ldots, n_{j}\right)=$ $p\left(n_{1}, \ldots, n_{j}: A_{1}, \ldots, A_{j}\right)$, we can easily verify that

$$
f\left(x_{1}, \ldots, x_{j}\right)=\sum_{n_{1}=0}^{\infty} \cdots \sum_{n_{j}=0}^{\infty} p\left(n_{1}, \ldots, n_{j}\right) e^{-n_{1} x_{1}-\ldots-n_{j} x_{j}} .
$$

We assume for convenience throughout this note that $n_{1} \leqslant \cdots \leqslant n_{j}$. The definition of $p\left(n_{1}, \ldots, n_{j}\right)$ is such that this assumption involves no loss of generality in the asymptotic results obtained.

When $|t|<2 \pi$, we have

$$
t e^{c t}\left(e^{t}-1\right)^{-1}=\sum_{\nu=0}^{\infty} B_{\nu}(c) t^{\nu} / \nu !
$$

for all $c$, where the $B_{\nu}(c) / \nu$ ! are the Bernouilli polynomials in $c$. From pp. 521523 of Knopp [4], we see that, if we write $P_{1}(t)=t-[t]-1 / 2$, then

$$
P_{1}(t)=B_{1}(t)=-\sum_{r=1}^{\infty}(r \pi)^{-1} \sin 2 r \pi t
$$

for $0<t<1$, and if we write

$$
\begin{gathered}
P_{2 \nu}(t)=2(-1)^{\nu-1} \sum_{r=1}^{\infty}(2 r \pi)^{-2 v} \cos 2 r \pi t, \\
P_{2 \nu+1}(t)=2(-1)^{\nu-1} \sum_{r=1}^{\infty}(2 r \pi)^{-2 \nu-1} \sin 2 r \pi t
\end{gathered}
$$

for all $\nu \geqslant 1$, then $P_{\nu}(t)=B_{\nu}(t) / \nu$ ! for $0<t<1$. Clearly every $P_{\nu}(t)$ is bounded, has period 1 and, for all $\nu>1, D P_{\nu}(t)=P_{\nu-1}(t) . B_{2 \nu}(0)$ for $\nu \geqslant 1$ are the Bernouilli numbers. For $\nu \geqslant 1, B_{2 v+1}(0)=0$ and $(2 \pi)^{2 \nu} B_{2 \nu}(0)=2(2 v) ! \zeta(2 v)$.

We define

$$
\begin{aligned}
\Lambda(t) & =\Lambda_{h(1) \ldots h(j)}\left(t: x_{1}, \ldots, x_{j}\right)=t^{-1} \prod_{l=1}^{\prime}\left(e^{M x_{l} t}-1\right)^{-1} e^{\left(M-a_{l h(l)}\right) x_{l} t} \\
& =t^{-1} \sum_{\nu_{1}=0}^{\infty} \cdots \sum_{\nu_{j}=0}^{\infty} \exp \left(-t \sum_{l=1}^{j}\left(M \nu_{l}+a_{l h(l)}\right) x_{l}\right), \\
F\left(x_{1}, \ldots, x_{j}\right) & =\sum_{l=1}^{j} n_{l} x_{l}+\sum_{r=1}^{\infty} \sum_{h(1)=1}^{q(1)} \ldots \sum_{h(j)=1}^{q(j)} \Lambda_{h(1) \ldots h(j)}\left(r: x_{1}, \ldots, x_{j}\right) .
\end{aligned}
$$


We put $X=x_{1} \cdots x_{j}$ and $x^{*}$ for the $x_{l}$ with maximum modulus, i.e. $\left|x^{*}\right|=$ $\max \left|x_{l}\right|$. Hence, for $|t|<2 \pi / M\left|x^{*}\right|$, we obtain from (2.3)

$$
\begin{aligned}
X M^{j} t^{j+1} \Lambda(t) & =\prod_{l=1}^{j} M x_{l} t\left(e^{M x_{l} t}-1\right)^{-1} e^{\left(M-a_{l n}(l) x_{l} t\right.} \\
& =\prod_{l=1}^{j} \sum_{\nu=0}^{\infty} B_{\nu}\left(\frac{1-a_{l h(l)}}{M}\right) \frac{\left(M x_{l} t\right)^{\nu}}{\nu !}=\sum_{m=0}^{\infty} Q_{m} t^{m}
\end{aligned}
$$

where $Q_{m}=Q_{m}\left(x_{1}, \ldots, x_{j}\right)$ are homogeneous polynomials of degree $m$ in $x_{1}, \ldots, x_{j}$. We write

$$
G(t)=X M^{j} \Lambda(t)-\sum_{m=0}^{j} Q_{m} t^{m-j-1}
$$

so that $G(t)=\sum_{m=j+1}^{\infty} Q_{m} t^{m-j-1}$ whenever $|t|<2 \pi|M| x^{*} \mid$, and we put

$$
\begin{aligned}
H & =H\left(x_{1}, \ldots, x_{j}\right) \\
& =\int_{0}^{\infty}\left\{X M^{j} \Lambda(t)-\sum_{m=0}^{j-1} Q_{m} t^{m-j-1}-Q_{j}\left(e^{t}-1\right)^{-1}\right\} d t .
\end{aligned}
$$

We write $z_{l}=x_{l} / x_{1}$ for $1 \leqslant l \leqslant j, Z=z_{2} \cdots z_{j}$ and

$$
U_{m}=U_{m}\left(z_{1}, \ldots, z_{j}\right)=M^{-m} Q_{m}\left(z_{1}, \ldots, z_{j}\right)
$$

for all $m \geqslant 0$. If we write

$$
\Omega(u)=\Omega\left(u: z_{2}, \ldots, z_{j}\right)=u^{-1} \prod_{l=1}^{j}\left(e^{z} l^{u}-1\right)^{-1} e^{(1-a \ln (l) / M) z} l^{u},
$$

then it follows from (2.4) that

$$
Z u^{j+1} \Omega(u)=\sum_{m=0}^{\infty} U_{m} u^{m}
$$

for $|u|<2 \pi /\left|z^{*}\right|$, where $z^{*}$ is the $z_{l}$ with maximum modulus. Observing that

$$
\begin{aligned}
\int_{0}^{\infty}\left\{\left(e^{t}-1\right)^{-1}\right. & \left.-M x_{1}\left(e^{M x_{1} t}-1\right)^{-1}\right\} d t \\
& =\left[\log \left\{\left(1-e^{-M x_{1} t}\right)^{-1}\left(1-e^{-t}\right)\right\}\right]_{0}^{\infty}=\log M x_{1},
\end{aligned}
$$

we substitute $u=M x_{1} t$ in (2.5) and an easy calculation gives

$$
H=X M^{j} I-Q_{j} \log M x_{1},
$$

where 
PARTITIONS OF LARGE MULTIPARTITES WITH CONGRUENCE CONDITIONS 303

$$
I=I\left(z_{2}, \ldots, z_{j}\right)=\int_{0}^{\infty} \beta\left(u: z_{2}, \ldots, z_{j}\right) d u
$$

$$
\beta\left(u: z_{2}, \ldots, z_{j}\right)=\Omega(u)-Z^{-1}\left\{\sum_{m=0}^{j-1} U_{m} u^{m-j-1}+U_{j}\left(e^{u}-1\right)^{-1}\right\} .
$$

For $s \geqslant 1$, let us write $Z^{\prime}=z_{2} \cdots z_{s}, Z^{\prime \prime}=z_{s+1} \cdots z_{j}$ and, for all $m \geqslant$ 0,

$$
\begin{aligned}
& V_{m}=V_{m}\left(z_{1}, \ldots, z_{s}\right)=M^{-m} Q_{m}\left(z_{1}, \ldots, z_{s}\right) \\
& W_{m}=W_{m}\left(z_{s+1}, \ldots, z_{j}\right)=M^{-m} Q_{m}\left(z_{s+1}, \ldots, z_{j}\right) .
\end{aligned}
$$

We define

$$
\begin{aligned}
& \Omega^{\prime}(u)=\Omega^{\prime}\left(u: z_{2}, \ldots, z_{s}\right)=u^{-1} \prod_{l=1}^{s}\left(e^{z} l^{u}-1\right)^{-1} e^{\left(1-a \ln (l)^{/ M) z} l^{u},\right.} \\
& \Omega^{\prime \prime}(u)=\Omega^{\prime \prime}\left(u: z_{s+1}, \ldots, z_{j}\right)=\prod_{i=s+1}^{j}\left(e^{z} l^{u}-1\right)^{-1} e^{(1-a \ln (l) / M) z} l^{u},
\end{aligned}
$$

and so, for $|u|<2 \pi /|z *|$,

$$
Z^{\prime} u^{s+1} \Omega^{\prime}(u)=\sum_{m=0}^{\infty} V_{m} u^{m}, \quad Z^{\prime \prime} u^{j-s} \Omega^{\prime \prime}(u)=\sum_{m=0}^{\infty} W_{m} u^{m} .
$$

It follows that, for all $m \geqslant 0$,

$$
U_{m}=\sum_{r=0}^{m} V_{r} W_{m-r}
$$

We define a generalization of the integral $I$ by writing

$$
I_{s r}^{\prime}=I_{s r}^{\prime}\left(z_{2}, \ldots, z_{s}\right)=\int_{0}^{\infty} \beta_{s r}^{\prime}\left(u: z_{2}, \ldots, z_{s}\right) d u,
$$

where, for $r \geqslant j+1$,

$$
\beta_{s r}^{\prime}\left(u: z_{2}, \ldots, z_{s}\right)=u^{r+s-j} \Omega^{\prime}(u)
$$

and, for $0 \leqslant r \leqslant j$,

$$
\begin{aligned}
\beta_{s r}^{\prime}\left(u: z_{2}, \ldots, z_{s}\right) & =u^{r+s-j} \Omega^{\prime}(u) \\
& -Z^{\prime-1}\left\{\sum_{m=0}^{j-r-1} V_{m} u^{m+r-j-1}+V_{j-r}\left(e^{u}-1\right)^{-1}\right\} .
\end{aligned}
$$

For $1 \leqslant l \leqslant j$, we write

$$
x_{l}=y_{l}+i \theta_{l}=y_{l}\left(1+i \xi_{l}\right)
$$


where $y_{l}>0$. We put

$$
\begin{aligned}
Y & =y_{1} \ldots y_{j}, \\
R_{m} & =R_{m}\left(y_{1}, \ldots, y_{j}\right)=Q_{m}\left(y_{1}, \ldots, y_{j}\right) \\
\bar{Q}_{m} & =\bar{Q}_{m}\left(x_{1}, \ldots, x_{j}\right)=\sum_{n(1)=1}^{q(1)} \ldots \sum_{n(j)=1}^{q(j)} Q_{m}\left(x_{1}, \ldots, x_{j}\right), \\
\bar{R}_{m} & =\bar{R}_{m}\left(y_{1}, \ldots, y_{j}\right)=\sum_{n(1)=1}^{q(1)} \ldots \sum_{n(j)=1}^{q(j)} R_{m}\left(y_{1}, \ldots, y_{j}\right), \\
\bar{H} & =\bar{H}\left(x_{1}, \ldots, x_{j}\right)=\sum_{n(1)=1}^{q(1)} \ldots \sum_{n(j)=1}^{q(j)} H\left(x_{1}, \ldots, x_{j}\right) .
\end{aligned}
$$

We let $y_{l} \sim \mu_{l}$, where the $\mu_{l}$ are defined by

$$
\mu_{l}=n_{l}^{-1}\left\{M^{-i} \zeta(j+1) \bar{R}_{0} n_{1} \cdots n_{j}\right\}^{1 /(j+1)} .
$$

Since $n_{1} \leqslant \cdots \leqslant n_{j}$, clearly $\mu_{1} \geqslant \cdots \geqslant \mu_{j}$.

From (6.2), which we prove later, we can easily deduce that a positive integer $\Delta$ can be found such that $\Delta$ is the smallest integer for which $\mu_{1}^{2 \Delta+j-1}=$ $o\left(\mu_{1} \cdots \mu_{j}\right)$. With this value of $\Delta$, we define

$$
F^{*}\left(x_{1}, \ldots, x_{j}\right)=\sum_{l=1}^{j} n_{l} x_{l}
$$

$$
+X^{-1} M^{-j}\left\{\sum_{m=0 ; m \neq j}^{2 \Delta+j-1} \zeta(j+1-m) \bar{Q}_{m}+\gamma \bar{Q}_{j}+\bar{H}\right\}
$$

For $1 \leqslant l \leqslant j$, we write $d_{l}$ for the greatest common divisor $\left(M, a_{l 1}-a_{l 2}\right.$, $\left.\ldots, a_{l 1}-a_{l q(l)}\right)$. Then we define $K=K\left(n_{1}, \ldots, n_{j}: A_{1}, \ldots, A_{j}\right)$ as the number of $\nu$ in $0 \leqslant \nu \leqslant M-1$ which satisfy the simultaneous congruences $n_{l} \equiv v a_{l 1}\left(\bmod d_{l}\right), 1 \leqslant l \leqslant j . \quad$ Clearly, since $d_{l}$ divides $a_{l 1}-a_{l n(l)}$ for $2<$ $h(l) \leqslant q(l), 1 \leqslant l \leqslant j$, these congruences are equivalent to $n_{l} \equiv v a_{l h}(l)\left(\bmod d_{l}\right)$ for any set of $h(1), \ldots, h(j)$. Finally, we write $q=\bar{Q}_{0}=\bar{R}_{0}=q(1) \cdots q(j)$.

3. Statement and proof of the main result. Employing the definitions of the last section, we can now state our principal result.

THEOREM 1. If, for $1 \leqslant l \leqslant j \quad(j>1)$, every $n_{l}$ tends to infinity subject to the condition that

$$
n_{1} \cdots n_{j}<n_{l}^{j+1-e_{1}}
$$

for any fixed positive number $\epsilon_{1}$, then 
PARTITIONS OF LARGE MULTIPARTITES WITH CONGRUENCE CONDITIONS 305

$$
\begin{aligned}
p\left(n_{1}, \ldots, n_{j}\right) \sim & K d_{1} \cdots d_{j} M^{-1}(j+1)^{-1 / 2}\left\{M^{j} / 2 \pi \zeta(j+1) q\right\}^{1 / 2 j} \\
& \times Y^{1 / 2(j+2)} e^{F^{*}\left(y_{1}, \ldots, y_{j}\right)}
\end{aligned}
$$

where the $y_{l}$ are functions of $\mu_{1}, \ldots, \mu_{j}$ such that $y_{l} \sim \mu_{l}$ and

$$
y_{l} \partial F^{*}\left(y_{1}, \ldots, y_{j}\right) / \partial y_{l}=o\left\{\left(\mu_{1} \cdots \mu_{j}\right)^{-y_{2}+\epsilon_{2}}\right\}
$$

for $1 \leqslant l \leqslant j$ and any fixed positive number $\epsilon_{2}$.

Proof. First we choose $\epsilon_{2}$ to satisfy

$$
0<2(j+1) \epsilon_{2}<\epsilon_{1}
$$

an inequality which will be used in the proof of Lemma 4. Next, we define $\eta$ and $\chi$ by

$$
\eta=\left(\mu_{1} \cdots \mu_{j}\right)^{1 / 2-\epsilon_{2}}, \quad \chi=\eta\left\{Y^{-1} M^{-j} \zeta(j+1) \bar{R}_{0}\right\}^{1 / 2} \sim C\left(n_{1} \cdots n_{j}\right)^{\epsilon_{2} /(j+1)} .
$$

From (2.2), we obtain

$$
\begin{aligned}
& p\left(n_{1}, \ldots, n_{j}\right) \\
& \quad=(2 \pi)^{-j} \int_{-\pi}^{\pi} \cdots \int_{-\pi}^{\pi} e^{n_{1} x_{1}+\ldots+n_{j} x_{j}+\log f\left(x_{1}, \ldots, x_{j}\right)} d \theta_{1} \cdots d \theta_{j} .
\end{aligned}
$$

Now, from (2.1),

$$
\begin{aligned}
\log f\left(x_{1}, \ldots, x_{j}\right)= & \sum_{\nu_{1}=0}^{\infty} \ldots \sum_{\nu_{j}=0}^{\infty} \sum_{h(1)=1}^{q(1)} \\
& \ldots \sum_{h(j)=1}^{q(j)} \sum_{r=1}^{\infty} r^{-1} \exp \left(-r \sum_{l=1}^{j}\left(M v_{l}+a_{l h(l)}\right) x_{l}\right) \\
= & \sum_{r=1}^{\infty} \sum_{h(1)=1}^{q(1)} \ldots \sum_{h(j)=1}^{q(j)} \Lambda_{h(1) \ldots h(j)}\left(r: x_{1}, \ldots, x_{j}\right)
\end{aligned}
$$

and so,

(3.5) $p\left(n_{1}, \ldots, n_{j}\right)=(2 \pi)^{-j} \int_{-\pi / M}^{\pi / M} \cdots \int_{-\pi / M}^{\pi / M} g\left(x_{1}, \ldots, x_{j}\right) d \theta_{1} \cdots d \theta_{j}$,

where, for $\omega=e^{2 \pi i / M}$, 


$$
\begin{aligned}
g\left(x_{1}, \ldots, x_{j}\right)= & e^{n_{1} x_{1}+\ldots+n_{j} x_{j}} \sum_{s_{1}=0}^{M-1} \ldots \sum_{s_{j}=0}^{M-1} \omega^{n_{1} s_{1}+\ldots+n_{j} s_{j}} \\
& \times \exp \left\{\sum_{r=1}^{\infty} \sum_{h(1)=1}^{q(1)} \cdots \sum_{h(j)=1}^{q(j)} \Lambda_{h(1) \ldots h(j)}\left(r: x_{1}, \ldots, x_{j}\right)\right. \\
& \left.\times \omega^{-r \Sigma_{l=1}^{j} \operatorname{lo} l(l)^{s} l}\right\} .
\end{aligned}
$$

In order to prove Theorem 1, we require an asymptotic expansion for the logarithm of the generating function $f\left(x_{1}, \ldots, x_{j}\right)$. The following result, which is of interest in itself, will be proved in $\$ 5$.

THEOREM 2. When $\left|\arg x_{l}\right|<1 / 2 \pi-\epsilon_{3}$ for $1 \leqslant l \leqslant j$,

$$
X M^{j} \log f\left(x_{1}, \ldots, x_{j}\right)=\sum_{m=0 ; m \neq j}^{2 k+j-1} \zeta(j+1-m) \bar{Q}_{m}+\gamma \bar{Q}_{j}+\bar{H}+O\left(x^{* 2 k+j}\right)
$$
as $x^{*} \longrightarrow 0$.

We also require the following lemmas.

LEMMA 1. An equivalence relation is defined on $\left\{\left(s_{1}, \ldots, s_{j}\right): 0 \leqslant s_{l} \leqslant\right.$ $M-1$ for $1 \leqslant l \leqslant j\}$ by setting $\left(s_{1}, \ldots, s_{j}\right)$ equivalent to $\left(s_{1}^{\prime}, \ldots, s_{j}^{\prime}\right)$ whenever $\Sigma_{1}^{j} a_{l h(l)} s_{l} \equiv \Sigma_{1}^{j} a_{l h(l)} s_{l}^{\prime}(\bmod M)$ for all $h(1), \ldots, h(j)$. Then

$$
\begin{aligned}
g\left(x_{1}, \ldots, x_{j}\right)= & e^{n_{1} x_{1}+\ldots+n_{j} x_{j}} K d_{1} \cdots d_{j} M^{-1} \sum^{*} \omega^{n_{1} s_{1}^{*}+\ldots+n_{j} s_{j}^{*}} \\
& \times \exp \left\{\sum_{r=1}^{\infty} \sum_{h(1)=1}^{q(1)} \ldots \sum_{h(j)=1}^{q(j)} \Lambda_{h(1) \ldots h(j)}\left(r: x_{1}, \ldots, x_{j}\right)\right. \\
& \left.\times \omega^{-r \Sigma_{l=1}^{j}{ }^{a} \ln (l)^{s}}\right\}
\end{aligned}
$$

where the sum $\Sigma^{*}$ is taken over a complete set of representatives of the equivalence classes.

LEMMA 2. If $\Sigma_{1}^{j}\left|\xi_{l}\right| \geqslant \eta$ and every $\left|\theta_{l}\right|<\pi / M$, then

$$
g\left(x_{1}, \ldots, x_{j}\right)=O\left\{\exp \left(F\left(y_{1}, \ldots, y_{j}\right)-C \chi^{2}\right)\right\}
$$

LEMMA 3. If $\Sigma_{1}^{j}\left|\xi_{l}\right| \leqslant \eta$, then 
PARTITIONS OF LARGE MULTIPARTITES WITH CONGRUENCE CONDITIONS 307

$$
\begin{aligned}
\sum^{(*)} \omega^{n_{1} s_{1}+\ldots+n_{j} s_{j}} \exp \left\{\sum_{r=1}^{\infty} \sum_{h(1)=1}^{q(1)} \ldots \sum_{h(j)=1}^{q(j)} \Lambda_{h(1) \ldots h(j)}\left(r: x_{1}, \ldots, x_{j}\right)\right. \\
\times \omega^{\left.-r \sum_{l=1}^{j} a_{l n}(l)^{s_{i}^{*}}\right\}} \\
=O\left\{\exp \left(F\left(y_{1}, \ldots, y_{j}\right)-\sum_{l=1}^{j} n_{l} y_{l}-C\left(\mu_{1} \cdots \mu_{j}\right)^{-1}\right)\right\},
\end{aligned}
$$

where the sum $\Sigma^{(*)}$ is taken over the same set as $\Sigma^{*}$ except that the term corresponding to the equivalence class where every $\Sigma_{1}^{j} a_{l n(l)} s_{l} \equiv 0(\bmod M)$ is omitted.

Lemma 4. If $\Sigma_{1}^{j}\left|\xi_{l}\right| \leqslant \eta$, then

$$
\begin{aligned}
F\left(x_{1}, \ldots, x_{j}\right)= & F^{*}\left(y_{1}, \ldots, y_{j}\right) \\
& -Y^{-1} M^{-j} \zeta(j+1) \bar{R}_{0}\left\{\sum_{l=1}^{j} \xi_{l}^{2}+\sum_{l=1}^{j-1} \sum_{m=l+1}^{j} \xi_{l} \xi_{m}\right\}+o(1) .
\end{aligned}
$$

Lemma 5. It is always possible to choose $y_{1}, \ldots, y_{j}$ so that $y_{l} \sim \mu_{l}$ and (3.2) holds for $1 \leqslant l \leqslant j$.

From (3.5), (3.6) and Lemma 1, it follows that

$$
\begin{aligned}
& M(2 \pi)^{j} \frac{p\left(n_{1}, \ldots, n_{j}\right)}{K d_{1} \cdots d_{j}}
\end{aligned}
$$

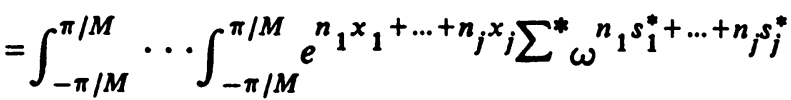

$$
\begin{aligned}
& \times \exp \left\{\sum_{r=1}^{\infty} \sum_{h(1)=1}^{q(1)} \ldots \sum_{h(j)=1}^{q(j)} \Lambda_{h(1) \ldots h(j)}\left(r: x_{1}, \ldots, x_{j}\right)\right. \\
& \left.\times \omega^{-r \Sigma_{l=1}^{j} a \ln (l)^{s} i}\right\} d \theta_{1} \cdots d \theta_{j}
\end{aligned}
$$

and, by Lemmas 2 and 3 , this is equal to

$$
\begin{aligned}
& \int_{\Sigma\left|\xi_{l}\right|<\eta} \ldots \int e^{F\left(x_{1}, \ldots, x_{j}\right)} d \theta_{1} \cdots d \theta_{j}+O\left\{e^{F\left(y_{1}, \ldots, y_{j}\right)-C x^{2}}\right\} \\
& +O\left\{\eta^{j} e^{F\left(y_{1}, \cdots, y_{j}\right)-C / \mu_{1} \cdots \mu_{j}}\right\} \text {. }
\end{aligned}
$$

By Lemma 4, the latter integral is asymptotic to 


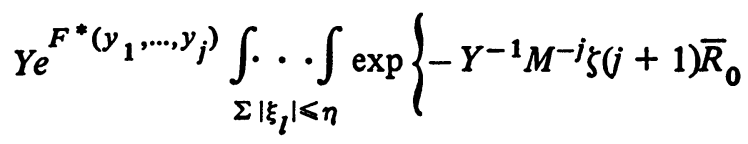

$$
\begin{aligned}
& \left.\times\left(\sum_{l=1}^{j} \xi_{l}^{2}+\sum_{l=1}^{j-1} \sum_{m=l+1}^{j} \xi_{l} \xi_{m}\right)\right\} d \xi_{1} \cdots d \xi_{j} \cdot
\end{aligned}
$$

If we transform the variables in this integral by writing

$$
\xi_{l}=\left\{Y M^{j} / \zeta(j+1) \bar{R}_{0}\right\}^{1 / 2} u_{l} \text { for } 1 \leqslant l \leqslant j
$$

we obtain

$$
\begin{aligned}
M(2 \pi)^{j} p\left(n_{1}, \ldots, n_{j}\right) / K d_{1} \cdots d_{j} \sim Y^{1 / 2(j+2)}\left\{M^{j} / \zeta(j+1) \bar{R}_{0}\right\}^{1 / 2 j} \\
\quad \times e^{F^{*}\left(y_{1}, \ldots, y_{j}\right)} \int_{\substack{\Sigma\left|u_{l}\right|<x \\
.}} \exp \left(-\sum u_{l}^{2}-\sum \sum u_{l} u_{m}\right) d u_{1} \cdots d u_{j}
\end{aligned}
$$

Since the integrand is positive everywhere,

$$
\begin{aligned}
\int_{-x / j}^{x / j} \cdots \int_{-x \mid j}^{x / j} & <\int_{\Sigma\left|u_{l}\right| \leqslant x} \cdots \int_{-x}^{x} \cdots \int_{-x}^{x} \exp \left(-\sum u_{l}^{2}-\sum u_{l} u_{m}\right) d u_{1} \cdots d u_{j} \\
& <\int_{-x}^{x} \cdots
\end{aligned}
$$

and so, by Lemma 4 of [10],

$$
\begin{aligned}
p\left(n_{1}, \ldots, n_{j}\right) \sim & K d_{1} \cdots d_{j} M^{-1}(j+1)^{-1 / 2}\left\{M^{j} / 2 \pi \zeta(j+1) \bar{R}_{0}\right\}^{1 / 2 j} \\
& \times Y^{1 / 2(j+2)} e^{F^{*}\left(y_{1}, \ldots, y_{j}\right)} .
\end{aligned}
$$

Theorem 1 follows from this and Lemma 5.

In order to examine $F^{*}\left(y_{1}, \ldots, y_{j}\right)$ more precisely, it is necessary to investigate $I$ and, for this purpose, we require two further lemmas.

LEMMA 6. If $g(t)$ and all its derivatives are continuous in $0 \leqslant t \leqslant 1$, then, for any $\tau$ satisfying $0<\tau<1$,

$$
g(\tau)=\int_{0}^{1} g(t) d t+\sum_{r=1}^{k} P_{r}(\tau)\left\{D^{r-1} g(1)-D^{r-1} g(0)\right\}-\sigma_{k}
$$

for all $k \geqslant 1$, where

$$
\sigma_{k}=\int_{0}^{1} P_{k}(\tau-t) D^{k} g(t) d t
$$


PARTITIONS OF LARGE MULTIPARTITES WITH CONGRUENCE CONDITIONS 309

Proof. For $r>1, P_{r}(t)$ is continuous, $D P_{r}(\tau-t)=-P_{r-1}(\tau-t)$ and $P_{r}(\tau-1)=P_{r}(\tau)$. Therefore, by integration by parts,

$$
\sigma_{r}=P_{r}(\tau)\left\{D^{r-1} g(1)-D^{r-1} g(0)\right\}+\sigma_{r-1}
$$

for all $r>1$. Also, $P_{1}(-0)-P_{1}(+0)=1, D P_{1}(\tau-t)=-1$ for all $t \neq \tau$ and $P_{1}(\tau-1)=P_{1}(\tau)$.

Now,

$$
\begin{aligned}
& \int_{0}^{\tau} D P_{1}(\tau-t) g(t) d t=P_{1}(+0) g(\tau)-P_{1}(\tau) g(0)-\int_{0}^{\tau} P_{1}(\tau-t) D g(t) d t \\
& \int_{\tau}^{1} D P_{1}(\tau-t) g(t) d t=P_{1}(\tau) g(1)-P_{1}(-0) g(\tau)-\int_{\tau}^{1} P_{1}(\tau-t) D g(t) d t
\end{aligned}
$$

and so,

$$
\begin{aligned}
g(\tau)-\int_{0}^{1} g(t) d t & =\left\{P_{1}(-0)-P_{1}(+0)\right\} g(\tau)+\int_{0}^{1} D P_{1}(\tau-t) g(t) d t \\
& =P_{1}(\tau)\{g(1)-g(0)\}-\int_{0}^{1} P_{1}(\tau-t) D g(t) d t
\end{aligned}
$$

The lemma follows easily from (3.7).

COROllary. For $|\arg v|<1 / 2 \pi-\epsilon_{4}, 0 \leqslant \tau<1$, and any fixed positive integer $k \geqslant 2$,

$$
v e^{v \tau}\left(e^{v}-1\right)^{-1}=1+\sum_{r=1}^{k-1} P_{r}(\tau) v^{r}+O\left(v^{k}\right)
$$

where the constant implied in the order term depends upon $\tau, k$ but is independent of $v$.

Proof. For $0<\tau<1$, putting $g(t)=e^{v t}$ in Lemma 6, we obtain

$$
e^{v \tau}=v^{-1}\left(e^{v}-1\right)+\sum_{r=1}^{k} P_{r}(\tau) v^{r-1}\left(e^{v}-1\right)-v^{k} \int_{0}^{1} P_{k}(\tau-t) e^{v t} d t .
$$

Since $v\left(e^{\operatorname{Re}(v)}-1\right) /\left(e^{v}-1\right) \operatorname{Re}(v)$ is bounded, it follows that

$$
v e^{v \tau}\left(e^{v}-1\right)^{-1}=1+\sum_{r=1}^{k-1} P_{r}(\tau) v^{r}+O\left(v^{k}\right) \text {. }
$$

It is well known that this formula also holds for $\tau=0$. (See, for example, [4, pp. 534-535].)

LEMMA 7. If $\left|\arg z_{l}\right|<1 / 2 \pi-\epsilon_{5}$ for $2 \leqslant l \leqslant j$, then

$$
Z^{\prime \prime} I\left(z_{2}, \ldots, z_{j}\right)=\sum_{r=0}^{k-1} I_{s r}^{\prime} W_{r}+\sum_{r=k}^{(j-s) k} O\left(I_{s r}^{\prime} z^{\prime \prime} \eta\right.
$$

for all $k>j$. 
Proof. From (2.7) and the preceding corollary,

$$
\begin{aligned}
& \left.\beta\left(u: z_{2}, \ldots, z_{j}\right)=Z^{\prime \prime-1} u^{s-j-1} \prod_{l=1}^{s}\left(e^{z} l^{u}-1\right)^{-1} e^{(1-a} \ln (l) / M\right) z l^{u} \\
& \times\left\{\sum_{r=0}^{k-1} w_{r} u^{r}+\sum_{r=k}^{(j-s) k} O\left(z^{\prime \prime r} u^{r}\right)\right\} \\
& -Z^{-1}\left\{\sum_{m=0}^{j-1} \sum_{r=0}^{m} V_{r} W_{m-r} u^{m-j-1}+\sum_{r=0}^{J} V_{r} W_{j-r}\left(e^{u}-1\right)^{-1}\right\} \\
& =Z^{\prime \prime}-1\left\{\sum_{r=0}^{k-1} \beta_{s r}^{\prime}\left(u: z_{2}, \ldots, z_{s}\right) W_{r}\right. \\
& \left.+\sum_{r=k}^{(j-s) k} O\left\{\beta_{s r}^{\prime}\left(u: z_{2}, \ldots, z_{s}\right) z^{\prime \prime r}\right\}\right\}
\end{aligned}
$$

The lemma follows by integration over $u$ from 0 to $\infty$.

Since $e^{t}-1>t^{m} / m$ ! for all positive $t$ and all positive integers $m$, we have, for all $r \geqslant j+1$,

$$
\beta_{s r}^{\prime}\left(u: y_{2} / y_{1}, \ldots, y_{s} / y_{1}\right)=O\left\{y_{1}^{s}\left(y_{1} \cdots y_{s}\right)^{-1}\right\}
$$

for $0 \leqslant u \leqslant 1 / 2 \pi$ and

$$
\beta_{s r}^{\prime}\left(u: y_{2} / y_{1}, \ldots, y_{s} / y_{1}\right)=O\left\{y_{1}^{s}\left(y_{1} \cdots y_{s}\right)^{-1} u^{-2}\right\}
$$

for $u \geqslant 1 / 2 \pi$. It follows that, for all $r \geqslant j+1$,

$$
I_{s r}^{\prime}\left(y_{2} / y_{1}, \ldots, y_{s} / y_{1}\right)=O\left\{\mu_{1}^{s}\left(\mu_{1} \cdots \mu_{s}\right)^{-1}\right\}
$$

From (2.4),

$$
\begin{aligned}
\sum_{m=0}^{\infty} V_{m} & \left(1, \frac{y_{2}}{y_{1}}, \ldots, \frac{y_{s}}{y_{1}}\right) u^{m} \\
& =\prod_{l=1}^{s}\left(e^{u y_{l} / y_{1}}-1\right)^{-1} e^{\left(1-a_{l n}(l) / M\right) u y_{l} / y_{1}} \frac{u y_{l}}{y_{1}}
\end{aligned}
$$

and so,

$$
\left|V_{m}\left(1, y_{2}\left|y_{1}, \ldots, y_{s}\right| y_{1}\right)\right| \pi^{m} \leqslant e^{s \pi}\left\{1+\frac{\pi}{2}+\sum_{\nu=1}^{\infty} B_{2 \nu}(0) \pi^{2 v} /(2 v) !\right\}=C \text {. }
$$

Therefore, for $0 \leqslant r \leqslant j$ and $0 \leqslant u \leqslant 1 / 2 \pi$, 


$$
\begin{aligned}
& \beta_{s r}^{\prime}\left(u: y_{2} / y_{1}, \ldots, y_{s} / y_{1}\right) \\
& =y_{1}^{s}\left(y_{1} \cdots y_{s}\right)^{-1}\left\{V_{j-r}\left(1, \frac{y_{2}}{y_{1}}, \ldots, \frac{y_{s}}{y_{1}}\right)\left\{u^{-1}-\left(e^{u}-1\right)^{-1}\right\}\right. \\
& \left.\quad+\sum_{m=j-r+1}^{\infty} V_{m}\left(1, \frac{y_{2}}{y_{1}}, \ldots, \frac{y_{s}}{y_{1}}\right) u^{m+r-j-1}\right\}
\end{aligned}
$$

and so,

$$
\begin{aligned}
\int_{0}^{1 / 2 \pi} \beta_{s r}^{\prime}\left(u: \frac{y_{2}}{y_{1}}, \ldots, \frac{y_{s}}{y_{1}}\right) d u & =O\left\{y_{1}^{s}\left(y_{1} \cdots y_{s}\right)^{-1} \sum_{m=0}^{\infty} 2^{-m}\right\} \\
& =O\left\{\mu_{1}^{s}\left(\mu_{1} \cdots \mu_{s}\right)^{-1}\right\} .
\end{aligned}
$$

Also, for $0 \leqslant r \leqslant j$ and $u \geqslant 1 / 2 \pi$,

$$
\beta_{s r}^{\prime}\left(u: y_{2} / y_{1}, \ldots, y_{s} / y_{1}\right)=O\left\{y_{1}^{s}\left(y_{1} \cdots y_{s}\right)^{-1} u^{-2}\right\}
$$

and so,

$$
\int_{y_{2} \pi}^{\infty} \beta_{s r}^{\prime}\left(u: y_{2} / y_{1}, \ldots, y_{s} / y_{1}\right) d u=O\left\{\mu_{1}^{s}\left(\mu_{1} \cdots \mu_{s}\right)^{-1}\right\} .
$$

Hence, for $0 \leqslant r \leqslant j$,

$$
I_{s r}^{\prime}\left(y_{2} / y_{1}, \ldots, y_{s} / y_{1}\right)=O\left\{\mu_{1}^{s}\left(\mu_{1} \cdots \mu_{s}\right)^{-1}\right\} .
$$

Now, if $\mu_{s+1}, \ldots, \mu_{j}$ are each $O\left(\mu_{1}^{1+\epsilon_{6}}\right)$ for some fixed positive number $\epsilon_{6}$ but none of $\mu_{2}, \ldots, \mu_{s}$ is $O\left(\mu_{1}^{1+\epsilon_{7}}\right)$ for any fixed positive number $\epsilon_{7}$, then it follows from Lemma 7 that

$$
\begin{aligned}
I\left(\frac{y_{2}}{y_{1}}, \ldots, \frac{y_{j}}{y_{1}}\right)= & y_{1}^{j-s}\left(y_{s+1} \cdots y_{j}\right)^{-1} \sum_{r=0}^{k-1} I_{s r}^{\prime}\left(\frac{y_{2}}{y_{1}}, \ldots, \frac{y_{s}}{y_{1}}\right) \\
& \times\left(M y_{1}\right)^{-r} Q_{r}\left(y_{s+1}, \ldots, y_{j}\right)+O\left\{\mu_{1}^{j-k}\left(\mu_{1} \cdots \mu_{j}\right)^{-1} \mu_{s+1}^{k}\right\} .
\end{aligned}
$$

If $k$ is chosen so that $\mu_{1}^{j-k}\left(\mu_{1} \cdots \mu_{f}\right)^{-1} \mu_{s+1}^{k}=o(1)$, then the expression for $I$ given in (3.8) may be used to calculate $F^{*}\left(y_{1}, \ldots, y_{j}\right)$ in Theorem 1.

4. Proofs of the first three lemmas.

Proof of Lemma 1. It is easily verified that the relation is an equivalence relation. We observe that the final exponential expression in (3.6) is the same for all $\left(s_{1}, \ldots, s_{j}\right)$ for which the sums $\Sigma a_{l h(l)^{s_{l}}}$ are congruent modulo $M$ for all $h(1), \ldots, h(j)$. To evaluate $g\left(x_{1}, \ldots, x_{j}\right)$ therefore, we first compute the 
sums $\Sigma^{\prime} \omega^{n_{1}} s_{1}+\ldots+n_{j} s^{\prime} j$, where each sum $\Sigma^{\prime}$ is taken over all the $\left(s_{1}, \ldots, s_{j}\right)$ for which $\Sigma a_{l h(l) s_{l}} \equiv \rho_{h(1) \ldots h(j)}(\bmod M)$ for all $h(1), \ldots, h(j)$ and the $\rho_{h(1) \ldots h(j)}$ are nonnegative integers less than $M$.

For any particular set of $\rho_{h(1) \ldots h(j)}$, for which there is no $\left(s_{1}, \ldots, s_{f}\right)$ such that $\Sigma a_{l h(l)} s_{l} \equiv \rho_{h(1) \ldots h(j)}(\bmod M)$ for all $h(1), \ldots, h(j)$, then clearly the sum is zero. Otherwise, there is at least one $\left(s_{1}, \ldots, s_{j}\right)$, say $\left(s_{1}^{*}, \ldots, s_{j}^{*}\right)$, such that $\Sigma a_{l h(l)} s_{l}^{*} \equiv \rho_{h(1) \ldots h())}(\bmod M)$ for all $h(1), \ldots, h(j)$. It follows that, for any $\left(s_{1}, \ldots, s_{j}\right)$ for which $\Sigma a_{l h(l)} s_{l} \equiv \rho_{h(1) \ldots h(j)}(\bmod M)$ for all $h(1), \ldots, h(j)$, then $\Sigma a_{l h(l)}\left(s_{l}-s_{l}^{*}\right) \equiv 0(\bmod M)$ for all $h(1), \ldots, h(j)$. These congruences are equivalent to the $j+1$ congruences $\Sigma a_{l 1}\left(s_{l}-s_{l}^{*}\right) \equiv 0(\bmod M)$ and $d_{h}\left(s_{h}-s_{h}^{*}\right) \equiv 0(\bmod M)$ for $1 \leqslant h \leqslant j$. Hence, in this case,

$$
\begin{aligned}
& \sum_{\omega^{\prime}}^{\prime}{ }^{n_{1} s_{1}+\ldots+n_{j} s_{j}}=\omega^{n_{1} s_{1}^{*}+\ldots+n_{j} s_{j}^{*}} \sum_{s_{1}=0}^{d_{1}-1} \ldots \sum_{s_{j}=0}^{d_{j}-1} \omega^{M\left(n_{1} s_{1} / d_{1}+\ldots+n_{j} s_{j} / d_{j}\right)} \\
& \Sigma M a_{l 1} s_{l} / d_{l} \equiv 0(\bmod M) \\
& =\omega^{n_{1} s_{1}^{*}+\ldots+n_{j} s_{j}^{*}} \sum_{s_{1}=0}^{d_{1}-1} \ldots \sum_{s_{j}=0}^{d_{j}-1} \omega^{M\left(n_{1} s_{1} / d_{1}+\ldots+n_{j} s_{j} / d_{j}\right)} \\
& \times M^{-1} \sum_{\nu=0}^{M-1} \omega^{-\nu \Sigma M a_{l 1} l_{l} / a_{l}} \\
& =\omega^{n_{1} s_{1}^{*}+\ldots+n_{j} s_{j}^{*}} M^{-1} \sum_{\nu=0}^{M-1} \sum_{s_{1}=0}^{d_{1}-1} \omega^{M s_{1}\left(n_{1}-\nu a_{1}\right) / d_{1}} \\
& \ldots \sum_{s_{j}=0}^{d_{j}-1} \omega^{M s_{j}\left(n_{j}-\nu a_{j 1}\right) / d_{j}} \\
& =\omega^{n_{1} s_{i}^{*}+\ldots+n_{j} s_{j}^{*}} M^{-1} d_{1} \cdots d_{j} K .
\end{aligned}
$$

Lemma 1 follows immediately.

Proof of Lemma 2. At least one $\xi_{l}$ satisfies $\left|\xi_{l}\right|>\eta / j$. Then

$$
\begin{aligned}
\left|e^{M x_{l}}-1\right|^{2} & =e^{2 M y_{l}}+1-2 e^{M y_{l}} \cos M y_{l} \xi_{l} \\
& =\left(e^{M y_{l}}-1\right)^{2}+4 e^{M y_{l}} \sin ^{2} y_{2} M \theta_{l} \geqslant\left(e^{M y_{l}}-1\right)^{2}\left(1+C \eta^{2}\right)
\end{aligned}
$$

for $\left|\theta_{l}\right| \leqslant \pi / M$. Hence, for any $h(1), \ldots, h(j)$,

$$
\left|\Lambda_{h(1) \ldots h(j)}\left(1: x_{1}, \ldots, x_{j}\right)\right| \leqslant\left(1-C \eta^{2}\right) \Lambda_{h(1) \ldots h(j)}\left(1: y_{1}, \ldots, y_{j}\right) .
$$


Also, for all $r>1$ and any $h(1), \ldots, h(j)$,

$$
\left|\Lambda_{h(1) \ldots h(j)}\left(r: x_{1}, \ldots, x_{j}\right)\right| \leqslant \Lambda_{h(1) \ldots h(j)}\left(r: y_{1}, \ldots, y_{j}\right)
$$

and so, from (3.6),

$$
\lg \left(x_{1}, \ldots, x_{j}\right) \mid \leqslant C \exp \left\{F\left(y_{1}, \ldots, y_{j}\right)-C \chi^{2}\right\}
$$

Proof of Lemma 3. Each of the terms in $\Sigma^{(*)}$ has at least one of the sums $\Sigma a_{l h(l)} s_{l}^{*} \neq \equiv 0(\bmod M)$. Then, for some $h(1), \ldots, h(j), \Sigma a_{l h(l)} s_{l}^{*} \equiv \rho$ $\not \equiv 0(\bmod M)$. Therefore,

$$
\begin{aligned}
& \Lambda_{h(1) \ldots h(j)}\left(1: x_{1}, \ldots, x_{j}\right) \omega^{-\Sigma a_{l h(l)} s_{l}^{*}} \\
& \quad=\Lambda_{h(1) \ldots h(j)}\left(1: y_{1}, \ldots, y_{j}\right)\{1+O(\eta)\}(\cos 2 \pi \rho / M-i \sin 2 \pi \rho / M)
\end{aligned}
$$

It follows that

$$
\begin{aligned}
& \left|\exp \left\{\sum_{r=1}^{\infty} \sum_{h(1)=1}^{q(1)} \ldots \sum_{h(j)=1}^{q(j)} \Lambda_{h(1) \ldots h(j)}\left(r: x_{1}, \ldots, x_{j}\right) \omega^{-r \sum a_{l h(l)} s_{l}^{*}}\right\}\right| \\
& =\exp \operatorname{Re}\left\{\sum_{r=1}^{\infty} \sum_{h(1)=1}^{q(1)} \ldots \sum_{h(j)=1}^{q(j)} \Lambda_{h(1) \ldots h(j)}\left(r: x_{1}, \ldots, x_{j}\right) \omega^{-r \sum a} \ln (l)^{s_{i}^{*}}\right\} \\
& \leqslant \exp \left\{\sum_{r=1}^{\infty} \sum_{h(1)=1}^{q(1)} \ldots \sum_{h(j)=1}^{q(j)} \Lambda_{h(1) \ldots h(j)}\left(r: y_{1}, \ldots, y_{j}\right)\right. \\
& \left.-Y^{-1}(1-\cos 2 \pi \rho / M)\left\{1+O(\eta)+O\left(\mu_{1}\right)\right\}\right\} \\
& \leqslant \exp \left\{\sum_{r=1}^{\infty} \sum_{h(1)=1}^{q(1)} \cdots \sum_{h(j)=1}^{q(j)} \Lambda_{h(1) \ldots h(j)}\left(r: y_{1}, \ldots, y_{j}\right)-C Y^{-1}\right\} .
\end{aligned}
$$

Lemma 3 follows immediately.

5. Proof of Theorem 2. In order to prove Theorem 2, we first establish the following lemma.

LEMMA 8. As $t \rightarrow \infty$ through real positive values, $D^{k} G(t) \rightarrow 0$ for all $k \geqslant 0$ and, for all $k \geqslant 1$,

$$
\int_{0}^{\infty}\left|D^{k} G(t)\right| d t=O\left(x^{* j+k}\right)
$$

Proof. Obviously $G(t) \rightarrow 0$ as $t \rightarrow \infty$. Let $t_{0}=1 / 2 \pi / M\left|x^{*}\right|$. Now $D^{k} \Sigma_{0}^{j} Q_{m} t^{m-j-1} \rightarrow 0$ as $t \rightarrow \infty$ and 


$$
\begin{aligned}
\int_{t_{0}}^{\infty} \mid D^{k} \sum_{m=0}^{j} & Q_{m} t^{m-j-1} \mid d t \\
& \leqslant \int_{t_{0}}^{\infty} \sum_{m=0}^{j}\left|Q_{m}\right|(j+1-m) \cdots(j+k-m) t^{m-j-k-1} d t \\
& =\sum_{m=0}^{j}\left|Q_{m}\right|(j+1-m) \cdots(j+k-1-m) t_{0}^{m-j-k}=O\left(x^{* j+k}\right) .
\end{aligned}
$$

Since

$$
D\left\{\left(e^{M x_{l} t}-1\right)^{-1}\right\}=-M x_{l}\left\{\left(e^{M x_{l} t}-1\right)^{-1}+\left(e^{M x_{l} t}-1\right)^{-2}\right\},
$$

we see that $D^{k} \Lambda(t)$ is the sum of a finite number of terms of the form

$$
C t^{-a-1} \prod_{l=1}^{j}\left(M x_{l}\right)^{r_{l}}\left\{\left(M-a_{l n}(l) x_{l}\right\}^{s_{l}}\left(e^{M x_{l} t}-1\right)^{-\rho_{l}-1}\right.
$$

where $a \geqslant 0, r_{l} \geqslant \rho_{l} \geqslant 0, s_{l} \geqslant 0, a+\Sigma_{1}^{j}\left(r_{l}+s_{l}\right)=k$. Hence, $D^{k} \Lambda(t) \rightarrow 0$ as $t \rightarrow \infty$ and $\int_{t_{0}}^{\infty}\left|D^{k} \Lambda(t)\right| d t$ is dominated by the sum of a finite number of terms of the form

$$
\begin{aligned}
& C|X| \int_{t_{0}}^{\infty}\left\{\prod_{l=1}^{j}\left|x_{l}\right|^{r_{l}+s_{l}}\left|e^{M x_{l} t}-1\right|^{-\rho_{l}-1}\right\} t^{-a-1} d t \\
& \leqslant C|X| \int_{t_{0}}^{\infty}\left\{\prod_{l=1}^{j}\left|x_{l}\right|^{r_{l}+s_{l}}\left|x_{l} t\right|^{-\rho_{l}-1}\right\} t^{-a-1} d t \\
& \leqslant C\left\{\prod_{l=1}^{j}\left|x_{l}\right|^{r_{l}+s_{l}-\rho_{l}}\right\} t_{0}^{-j-a-\Sigma \rho_{l}} \\
& \leqslant C\left|x^{*}\right|^{\Sigma\left(r_{l}+s_{l}-\rho_{l}\right)}|x *|^{j+a+\Sigma \rho_{l}}=O\left(x^{* j+k}\right) \text {. }
\end{aligned}
$$

Therefore, $D^{k} G(t) \rightarrow 0$ as $t \rightarrow \infty$ and $\int_{t_{0}}^{\infty}\left|D^{k} G(t)\right| d t=O\left(x^{* j+k}\right)$.

By substituting $t=\pi / M\left|x^{*}\right|$ in (2.4), we obtain

$$
\sum_{m=0}^{\infty}\left|Q_{m}\right|\left(\pi / M\left|x^{*}\right|\right)^{m} \leqslant \prod_{l=1}^{j} e^{\pi}\left\{1+\frac{\pi}{2}+\sum_{\nu=1}^{\infty} B_{2 \nu}(0) \pi^{2 \nu} /(2 \nu) !\right\}=C
$$

and so, $\left|Q_{m}\right| \leqslant C\left(M\left|x^{*}\right| / \pi\right)^{m}$. Now, for $0 \leqslant t \leqslant t_{0}$,

$$
\left|D^{k} G(t)\right| \leqslant \sum_{m=j+k+1}^{\infty}(m-j-1) \cdots(m-j-k)\left|Q_{m}\right| t^{m-j-k-1}
$$

and hence, 
PARTITIONS OF LARGE MULTIPARTITES WITH CONGRUENCE CONDITIONS 315

$$
\begin{aligned}
\int_{0}^{t} 0\left|D^{k} G(t)\right| d t & \leqslant C \sum_{m=j+k+1}^{\infty}(m-j-1) \cdots(m-j-k+1) 2^{j+k-m}\left|x^{*}\right|^{j+k} \\
& =O\left(x^{* j+k}\right) .
\end{aligned}
$$

This completes the proof of the lemma.

Proof of Theorem 2. For any positive integer $N$, we have

$$
\begin{aligned}
\int_{0}^{N} P_{1}(t) D G(t) d t & =\sum_{r=0}^{N-1} \int_{0}^{1}(t-1 / 2) D G(t+r) d t \\
& =1 / 2\{G(0)+G(N)\}+\sum_{r=1}^{N-1} G(r)-\int_{0}^{N} G(t) d t .
\end{aligned}
$$

By repeated integration by parts, Lemma 8 shows that

$$
\begin{aligned}
\int_{0}^{\infty} P_{1}(t) D G(t) d t & =\sum_{s=1}^{2 k}(-1)^{s} P_{s+1}(0) D^{s} G(0)+\int_{0}^{\infty} P_{2 k+1}(t) D^{2 k+1} G(t) d t \\
& =\sum_{s=1}^{k-1}(-1)^{s} B_{2 s}(0) Q_{2 s+j} / 2 s+O\left(x^{* 2 k+j}\right) .
\end{aligned}
$$

Now,

$$
\sum_{r=1}^{N-1} G(r)-\int_{0}^{N} G(t) d t=A_{1}+A_{2}+A_{3},
$$

where

$$
\begin{aligned}
& A_{1}=\sum_{r=1}^{N-1}\left\{G(r)+Q_{j} / r\right\}, \\
& A_{2}=Q_{j}\left\{\int_{0}^{N}\left(t^{-1}-\left(e^{t}-1\right)^{-1}\right) d t-\sum_{r=1}^{N-1} r^{-1}\right\}, \\
& A_{3}=-\int_{0}^{N}\left\{G(t)+Q_{j}\left(t^{-1}-\left(e^{t}-1\right)^{-1}\right)\right\} d t .
\end{aligned}
$$

As $N \rightarrow \infty$,

$$
\begin{aligned}
& A_{1} \rightarrow X M^{j} \sum_{r=1}^{\infty} \Lambda(r)-\sum_{m=0}^{j-1} \zeta(j+1-m) Q_{m}, \\
& A_{2}=Q_{j}\left\{\log N-\log \left(1-e^{-N}\right)-\sum_{r=1}^{N-1} r^{-1}\right\} \rightarrow-\gamma Q_{j}, \\
& A_{3} \rightarrow-\int_{0}^{\infty}\left\{G(t)+Q_{j}\left(t^{-1}-\left(e^{t}-1\right)^{-1}\right)\right\} d t=-H .
\end{aligned}
$$


Therefore, letting $N \rightarrow \infty$ in (5.1), we obtain

$$
\int_{0}^{\infty} P_{1}(t) D G(t) d t=X M^{j} \sum_{r=1}^{\infty} \Lambda(r)-\sum_{m=0}^{j-1} \zeta(j+1-m) Q_{m}-\gamma Q_{j}+1 / 2 Q_{j+1}-H,
$$

and Theorem 2 follows from (3.4) and (5.2) since $\zeta(0)=-1 / 2$ and $\zeta(-2 s)=0$, $\zeta(1-2 s)=(-1)^{s} B_{2 s}(0) / 2 s$ for all positive integers $s$.

6. Proof of Lemma 4. We define the linear operator $T$ by

$$
\begin{aligned}
\operatorname{Th}\left(x_{1}, \ldots, x_{j}\right)= & h\left(x_{1}, \ldots, x_{j}\right)-h\left(y_{1}, \ldots, y_{j}\right) \\
& -\sum_{l=1}^{j} i \xi_{l} y_{l} \frac{\partial h}{\partial y_{l}}\left(y_{1}, \ldots, y_{j}\right),
\end{aligned}
$$

where $h\left(x_{1}, \ldots, x_{j}\right)$ is any function of $x_{1}, \ldots, x_{j}$ with continuous first order partial derivatives. First we prove that

(6.1) $T F^{*}\left(x_{1}, \ldots, x_{j}\right)=-Y^{-1} M^{-i} \zeta(j+1) \bar{R}_{0}\left(\sum_{l=1}^{j} \xi_{l}^{2}+\sum_{l=1}^{j-1} \sum_{m=l+1}^{j} \xi_{l} \xi_{m}\right)$

$$
+o(1) \text {. }
$$

Trivial calculations show that

$$
\begin{gathered}
T\left(\sum_{l=1}^{j} n_{l} x_{l}\right)=0 \\
T\left(X^{-1}\right)=-Y^{-1}\left(\sum_{l=1}^{j} \xi_{l}^{2}+\sum_{l=1}^{j-1} \sum_{m=l+1}^{j} \xi_{l} \xi_{m}\right)+O\left(\eta^{3} Y^{-1}\right) \\
T\left(X^{-1} Q_{j} \log x_{1}\right)=O\left(\eta^{2} Y^{-1} R_{j} \log y_{1}\right)
\end{gathered}
$$

and, for all $m>0$,

$$
T\left(X^{-1} Q_{m}\right)=O\left(\eta^{2} Y^{-1} R_{m}\right)
$$

Now

$$
\eta^{3} Y^{-1}=O\left\{\left(\mu_{1} \cdots \mu_{j}\right)^{1 / 2-3 \epsilon} 2\right\}=o(1) \text {. }
$$

From (3.1), $n_{1}^{j} \leqslant n_{1} \cdots n_{j}<n_{1}^{j+1-\epsilon_{1}}, n_{j}<n_{1}^{2-\epsilon_{1}}$, and therefore it follows from (2.8) that

Hence, we have

$$
C n_{1}^{-1+j \epsilon_{1} /(j+1)}<\mu_{j} \leqslant \mu_{1}<C n_{1}^{-\epsilon} /(j+1)
$$

$$
\mu_{j} \leqslant \mu_{1}<\mu_{j}^{\epsilon} /(j+1), \quad \log \mu_{j}=O\left(\log \mu_{1}\right) .
$$

Also, $\eta^{2} Y^{-1}\left|R_{j} \log y_{1}\right|$ and $\eta^{2} Y^{-1}\left|R_{m}\right|$ for all $m>0$ are each less than 
PARTITIONS OF LARGE MULTIPARTITES WITH CONGRUENCE CONDITIONS 317

$$
\begin{aligned}
C \mu_{1}\left(\mu_{1} \cdots \mu_{j}\right)^{-2 \epsilon_{2}} & =C n_{1}^{-1}\left(n_{1} \cdots n_{j}\right)^{\left(1+2 \epsilon_{2}\right) /(j+1)} \\
& <C n_{1}^{2 \epsilon_{2}-\epsilon_{1}\left(1+2 \epsilon_{2}\right) /(j+1)}=o(1)
\end{aligned}
$$

by (3.3). We have, therefore,

$$
\begin{gathered}
T\left(X^{-1}\right)=-Y^{-1}\left(\sum_{l=1}^{j} \xi_{l}^{2}+\sum_{l=1}^{j-1} \sum_{m=l+1}^{j} \xi_{l} \xi_{m}\right)+o(1), \\
T\left(X^{-1} Q_{j} \log x_{1}\right)=o(1)
\end{gathered}
$$

and, for all $m>0, T\left(X^{-1} Q_{m}\right)=o(1)$.

If we write $\Gamma(t)=h\left\{y_{1}\left(1+i \xi_{1} t\right), \ldots, y_{j}\left(1+i \xi_{j} t\right)\right\}$, where $h\left(x_{1}, \ldots, x_{j}\right)$ has continuous second order partial derivatives, then Taylor's theorem gives

$$
\Gamma(1)=\Gamma(0)+D \Gamma(0)+1 / 2 D^{2} \Gamma(\psi)
$$

for some $\psi$ which satisfies $0<\psi<1$. It follows that

$$
\begin{aligned}
& \operatorname{Th}\left(x_{1}, \ldots, x_{j}\right) \\
& \quad=-\frac{1}{2} \sum_{l=1}^{j} \sum_{m=1}^{j} \xi_{l} \xi_{m} y_{l} y_{m} \frac{\partial^{2} h}{\partial x_{l} \partial x_{m}}\left\{y_{1}\left(1+i \xi_{1} \psi\right), \ldots, y_{j}\left(1+i \xi_{j} \psi\right)\right\} .
\end{aligned}
$$

For all $l>1$, routine calculations give $\partial^{2} \Omega(u) / \partial x_{l}^{2}=\Omega(u)\left\{\left(a_{l n}(l){ }^{u / M x_{1}}+u / x_{1}\left(e^{z} l^{u}-1\right)\right)^{2}+u^{2} e^{z} l^{u} \mid x_{1}^{2}\left(e^{2} l^{u}-1\right)^{2}\right\}$, with similar expressions for the other second order partial derivatives. Since $e^{t}-1>t^{r} / r$ ! for all positive $t$ and all positive integers $r$, we have

$$
T \Omega(u)=O\left\{\eta^{2} \mu_{1}^{j}\left(\mu_{1} \ldots \mu_{j}\right)^{-1} u^{-j-1}\right\}
$$

and so,

$$
T\left\{\int_{1 / 2 \pi}^{\infty} \Omega(u) d u\right\}=O\left(\eta^{2} \mu_{1}^{j} / \mu_{1} \cdots \mu_{j}\right)
$$

If we substitute $t=\pi / M y_{1}$ in (2.4), we obtain

$$
\sum_{m=0}^{\infty} R_{m}^{*}\left(\pi / M y_{1}\right)^{m} \leqslant e^{j \pi}\left\{1+\frac{\pi}{2}+\sum_{\nu=1}^{\infty} B_{2 \nu}(0) \pi^{2 \nu} /(2 v) !\right\}^{\prime}=C,
$$

where $R_{m}^{*}$ is written for the sum of the moduli of the monomials of which the polynomial $R_{m}=Q_{m}\left(y_{1}, \ldots, y_{j}\right)$ is composed. Therefore, for all $m \geqslant 0$, 
$R_{m}^{*} y_{1}^{-m} \leqslant C M^{m} \pi^{-m}$. Since $Z^{-1} U_{m}$ is a sum of monomials of the form $C M^{-m} \Pi_{l=1}^{j}\left(x_{l} \mid x_{1}\right)^{r_{l}-1}$, where $\Sigma r_{l}=m$ and

$$
\sum_{l=1}^{j} \sum_{m=1}^{j} x_{l} x_{m} \frac{\partial^{2}}{\partial x_{l} \partial x_{m}}\left\{\prod_{l=1}^{j}\left(\frac{x_{l}}{x_{1}}\right)^{r_{l}-1}\right\}<j^{2} m^{2} \prod_{l=1}^{j}\left(\frac{x_{l}}{x_{1}}\right)^{r_{l}-1},
$$

it follows that

$$
\left|T\left(Z^{-1} U_{m}\right)\right| \leqslant C \eta^{2} m^{2} M^{-m} Y^{-1} R_{m}^{*} y_{1}^{j-m} \leqslant C \eta^{2} m^{2} \pi^{-m} y_{1}^{j} Y^{-1} .
$$

We can immediately deduce that

$$
\left.T\left(\int_{1 / 2 \pi}^{\infty} Z^{-1}\left\{\sum_{m=0}^{j-1} U_{m} u^{m-j-1}+U_{j}\left(e^{u}-1\right)^{-1}\right\} d u\right)=d \frac{\eta^{2} \mu_{1}^{j}}{\mu_{1} \cdots \mu_{j}}\right) .
$$

Also, since for $0 \leqslant u \leqslant 1 / 2 \pi$

$$
\beta\left(u: z_{2}, \ldots, z_{j}\right)=Z^{-1}\left\{\sum_{m=j+1}^{\infty} U_{m} u^{m-j-1}+U_{j}\left(u^{-1}-\left(e^{u}-1\right)^{-1}\right)\right\},
$$

we deduce that

$$
\begin{aligned}
T\left\{\int_{0}^{1 / 2 \pi} \beta\left(u: z_{2}, \ldots, z_{j}\right) d u\right\} & =O\left(\eta^{2} y_{1}^{j} Y^{-1} \sum_{m=0}^{\infty} m^{2} 2^{-m}\right) \\
& =O\left(\eta^{2} \mu_{1}^{j} / \mu_{1} \cdots \mu_{j}\right) .
\end{aligned}
$$

Combining the results of the last two paragraphs, we have

$$
T(I)=O\left(\eta^{2} \mu_{1}^{j} / \mu_{1} \cdots \mu_{j}\right)=O\left(n_{1}^{2 e_{2}-e_{1}\left(j+2 e_{2}\right) /(j+1)}\right)=O(1)
$$

by (3.3). (6.1) follows from (2.6) and (2.9). From (3.2), we have

$$
i \xi_{l} y_{l} \partial F^{*}\left(y_{1}, \ldots, y_{j}\right) / \partial y_{l}=o(1)
$$

for $1 \leqslant l \leqslant j$. Therefore, from (6.1),

$$
\begin{aligned}
F^{*}\left(x_{1}, \ldots, x_{j}\right)= & F^{*}\left(y_{1}, \ldots, y_{j}\right) \\
& -Y^{-1} M^{-i \zeta}(j+1) \bar{R}_{0}\left(\sum_{l=1}^{j} \xi_{l}^{2}+\sum_{l=1}^{i-1} \sum_{m=l+1}^{j} \xi_{l} \xi_{m}\right)+o(1) .
\end{aligned}
$$

Again, we see from (2.9), (3.4) and Theorem 2 that

$$
F\left(x_{1}, \ldots, x_{j}\right)=F^{*}\left(x_{1}, \ldots, x_{j}\right)+o(1)
$$

and Lemma 4 follows immediately. 
7. Proof of Lemma 5. As the proof of this lemma is very similar to that of Lemma 3 in [11], we provide only an outline. It is easily seen that, for all $l>1$,

$$
\begin{aligned}
x_{l} \partial \Omega(u) / \partial x_{l} & =\Omega(u)\left\{\left(1-a_{l n}(l)\right.\right. \\
& \left.\mid M) z_{l} u-z_{l} u e^{z} l^{u}\left(e^{z} l^{u}-1\right)^{-1}\right\} \\
& =-z_{l} \Omega(u)\left\{a_{\ln (l)} u / M+u\left(e^{z} l^{u}-1\right)^{-1}\right\} .
\end{aligned}
$$

Because of the uniqueness of power series expansions, this equality implies that, if both sides are expanded in increasing powers of $u$, the corresponding coefficients are equal. Therefore,

$$
x_{l} \partial I / \partial x_{l}=-z_{l}\left\{\left(a_{l n(l)} / M\right) I_{j 1}^{\prime}+I^{(l)}\right\}
$$

where

$$
I^{(l)}=I^{(l)}\left(z_{2}, \ldots, z_{j}\right)=I_{j+1,1}^{\prime}\left(z_{2}, \ldots, z_{j}, z_{l}\right)
$$

and the $a_{l h(l)}$ associated with the second $z_{l}$ is $M$. It follows immediately that

$$
\begin{aligned}
& y_{l} \partial I\left(y_{2} / y_{1}, \ldots, y_{j} / y_{1}\right) / \partial y_{l} \\
& \quad=-\left(y_{l} / y_{1}\right)\left\{\left(a_{l n}(l)\right.\right. \\
& \left.\quad M) I_{j 1}^{\prime}\left(y_{2} / y_{1}, \ldots, y_{j} / y_{1}\right)+I^{(l)}\left(y_{2} / y_{1}, \ldots, y_{j} / y_{1}\right)\right\} .
\end{aligned}
$$

Similarly,

$$
\begin{aligned}
& y_{1} \partial I\left(y_{2} / y_{1}, \ldots, y_{j} / y_{1}\right) / \partial y_{1} \\
& \quad=\sum_{k=2}^{j}\left(y_{k} / y_{1}\right)\left\{\left(a_{k h(k)} / M\right) I_{j 1}^{\prime}\left(y_{2} / y_{1}, \ldots, y_{j} / y_{1}\right)+I^{(k)}\left(y_{2} / y_{1}, \ldots, y_{j} / y_{1}\right)\right\} .
\end{aligned}
$$

Now, if $\mu_{s+1}, \ldots, \mu_{j}$ are each $O\left(\mu_{1}^{1+\epsilon_{6}}\right)$ for some fixed positive number $\epsilon_{6}$ but none of $\mu_{2}, \ldots, \mu_{s}$ is $O\left(\mu_{1}^{1+\epsilon}\right)$ for any fixed positive number $\epsilon_{7}$, then both $I_{j 1}^{\prime}\left(y_{2} / y_{1}, \ldots, y_{j} / y_{1}\right)$ and $I^{(l)}\left(y_{2} / y_{1}, \ldots, y_{j} / y_{1}\right)$ can be expanded in powers of $y_{s+1}, \ldots, y_{j}$ as $I\left(y_{2} / y_{1}, \ldots, y_{j} / y_{1}\right)$ was in (3.8). It follows from (2.9) that $Y y_{l} \partial F^{*}\left(y_{1}, \ldots, y_{j}\right) / \partial y_{l}$ can be written in the form

$$
\begin{aligned}
Y y_{l} n_{l} & +\sum_{k(1) \ldots k(j)} c_{k(1) \ldots k(j)}^{(l)} y_{1}^{k(1)} \cdots y_{j}^{k(j)} \\
& +y_{1}^{\prime} \sum_{k(s+1) \ldots k(j)} c_{k(s+1) \ldots k(j)}\left(\frac{y_{s+1}}{y_{1}}\right)^{k(s+1)} \cdots\left(\frac{y_{j}}{y_{1}}\right)^{k(j)} \\
& +o\left\{\left(\mu_{1} \cdots \mu_{j}\right)^{1 / 2+e_{2}}\right\}
\end{aligned}
$$

where the first sum is taken over all nonnegative integers $k(1), \ldots, k(j)$ such that $\mu_{1}^{k(1)} \cdots \mu_{j}^{k(j)}$ is not $o\left\{\left(\mu_{1} \cdots \mu_{j}\right)^{1 / 2+e_{2}}\right\}$ and the second sum is taken over 
all nonnegative integers $k(s+1), \ldots, k(j)$ such that $\left(\mu_{s+1} / \mu_{1}\right)^{k(s+1)} \ldots$ $\left(\mu_{j} / \mu_{1}\right)^{k(j)}$ is not $o\left\{\mu_{1}^{-j}\left(\mu_{1} \cdots \mu_{j}\right)^{1 / 2+\epsilon_{2}}\right\}$. The coefficients in the first sum are constants except when $k(1)+\cdots+k(j)=j$ in which case they are linear functions of $\log y_{1}$. The coefficients in the second sum are $O(1)$ and involve definite integrals of the form $I_{s r}^{\prime}\left(y_{2} / y_{1}, \ldots, y_{s} / y_{1}\right)$ and $I_{s+1, r}^{\prime}\left(y_{2} / y_{1}, \ldots, y_{s} / y_{1}, y_{l} / y_{1}\right)$ for $2 \leqslant l \leqslant s$. Also, for $1 \leqslant l \leqslant j$, we have

$$
c_{0 \ldots 0}^{(l)}=-M^{-j} \zeta(j+1) \bar{R}_{0} .
$$

We put

$$
\begin{array}{r}
y_{l}=\mu_{l} \sum_{k(1) \ldots k(j) k^{\prime}(s+1) \ldots k^{\prime}(j)} \gamma_{k(1) \ldots k(j) k^{\prime}(s+1) \ldots k^{\prime}(j)}^{(l)} \mu_{1}^{k(1)} \ldots \mu_{j}^{k(j)} \\
\times\left(\mu_{s+1} / \mu_{1}\right)^{k^{\prime}(s+1)} \ldots\left(\mu_{j} / \mu_{1}\right)^{k^{\prime}(j)}
\end{array}
$$

for $1 \leqslant l \leqslant j$, where the sum is taken over all nonnegative integers $k(1), \ldots$, $k(j), k^{\prime}(s+1), \ldots, k^{\prime}(j)$ such that $\mu_{1}^{k(1)} \ldots \mu_{j}^{k(j)}\left(\mu_{s+1} / \mu_{1}\right)^{k^{\prime}(s+1)} \ldots$ $\left(\mu_{j} / \mu_{1}\right)^{k^{\prime}(j)}$ is not $o\left\{\left(\mu_{1} \cdots \mu_{j}\right)^{1 / 2+\epsilon_{2}}\right\}$. From (2.8), $n_{l} \mu_{l}\left(\mu_{1} \cdots \mu_{j}\right)=$ $M^{-j} \zeta(j+1) \bar{R}_{0}$ and therefore, it is easily seen from (7.2) that, for all $l$, $\gamma_{0 . \ldots 0,0 \ldots 0}^{(l)}=1$. Hence, the expressions for $y_{l}$ given in (7.3) satisfy $y_{l} \sim \mu_{l}$.

Now, by substituting in (7.1) for each $y_{l}$ the finite series given in (7.3) and by equating the coefficients of $\mu_{1}^{k(1)} \cdots \mu_{j}^{k(j)}\left(\mu_{s+1} / \mu_{1}\right)^{k^{\prime}(s+1)} \ldots$ $\left(\mu_{j} / \mu_{1}\right)^{k^{\prime}(j)}$ on each side of the resulting equation, we can calculate the other coefficients successively. Thus, for example,

$$
2 \gamma_{10 \ldots 0,0 \ldots 0}^{(l)}+\sum_{\nu=1 ; \nu \neq l}^{j} \gamma_{10 \ldots 0,0 \ldots 0}^{(\nu)}=-c_{10 \ldots 0}^{(l)} M^{-j} \zeta(j+1) \bar{R}_{0}
$$

for $1 \leqslant l \leqslant j$ and these equations can easily be solved for each $\gamma_{10 \ldots 0,0 \ldots 0}^{(l)}$ in terms of the $c_{10 \ldots 0}^{(\nu)}$ for $1 \leqslant \nu \leqslant j$. Also, for any $k(1), \ldots, k(j), k^{\prime}(s+1), \ldots$, $k^{\prime}(j)$

$$
\begin{aligned}
2 \gamma_{k(1) \ldots k(j) k^{\prime}(s+1) \ldots k^{\prime}(j)}^{(l)} & +\sum_{\nu=1 ; \nu \neq l}^{j} \gamma_{k(1) \ldots k(j) k^{\prime}(s+1) \ldots k^{\prime}(j)}^{(\nu)} \\
& =c_{k(1) \ldots k(j) k^{\prime}(s+1) \ldots k^{\prime}(j)}^{*(l)},
\end{aligned}
$$

where the $c_{k(1) \ldots k(j) k^{\prime}(s+1) \ldots k^{\prime}(j)}^{*(l)}$ involve the coefficients $c_{k(1) \ldots k(j)}^{(l)}$, $c_{k(s+1) \ldots k(j)}^{\prime(l)}$ and the $\gamma_{\nu(1) \ldots \nu(j) \nu^{\prime}(s+1) \ldots \nu^{\prime}(j)}^{(l)}$ for which every $\nu(l) \leqslant k(l)$, every $\nu^{\prime}(l) \leqslant k^{\prime}(l)$ and $\Sigma_{1}^{j} \nu(l)+\Sigma_{s+1}^{j} \nu^{\prime}(l)<\Sigma_{1}^{j} k(l)+\Sigma_{s+1}^{j} k^{\prime}(l)$. These equations can be solved to give each $\gamma_{k(1) \ldots k(j) k^{\prime}(s+1) \ldots k^{\prime}(j)}^{(l)}$ in terms of the $c_{k}^{*}(\nu) \ldots k(j) k^{\prime}(s+1) \ldots k^{\prime}(j)$ for $1 \leqslant \nu \leqslant j$. Therefore, the coefficients $\gamma_{k(1) \ldots k(j) k^{\prime}(s+1) \ldots k^{\prime}(j)}^{(l)}$ can be calculated successively, provided that we calculate first the $\gamma_{k(1) \ldots k(j) k^{\prime}(s+1) \ldots k^{\prime}(j)}^{(l)}$ for which $\Sigma_{1}^{\prime} k(l)+\Sigma_{s+1}^{\prime} k^{\prime}(l)=1$, then 
those for which $\Sigma_{1}^{j} k(l)+\Sigma_{s+1}^{j} k^{\prime}(l)=2$, and so on. The $y_{l}$ can thus be expressed completely in terms of the $\mu_{l}$ and this completes the proof of Lemma 5 .

8. Concluding remarks. The dominant term in the asymptotic expansion for $p\left(n_{1}, \ldots, n_{j}\right)$ gives

$$
\left.\log p\left(n_{1}, \ldots, n_{j}\right) \sim(j+1)\left\{M^{-j} q(1) \cdots q(j)\right\}(j+1) n_{1} \cdots n_{j}\right\}^{1 /(j+1)},
$$

which generalizes the well-known results for $j=1$,

$$
\begin{array}{ll}
\log p\left(n_{1}: A_{1}\right) \sim \pi\left(2 n_{1} / 3\right)^{1 / 2}, & A_{1}=\{1,2,3, \ldots\}, \\
\log p\left(n_{1}: A_{1}\right) \sim \pi\left(n_{1} / 3\right)^{1 / 2}, & A_{1}=\{1,3,5, \ldots\} .
\end{array}
$$

When $\mu_{2}, \ldots, \mu_{j}$ are each $O\left(\mu_{1}^{1+\epsilon_{6}}\right)$ for some fixed positive number $\epsilon_{6}$, the asymptotic formula for $p\left(n_{1}, \ldots, n_{j}\right)$ is expressed entirely in terms of elementary functions. Otherwise, the formula involves the definite integrals $I_{s r}^{\prime}$, which we have been unable to express in terms of elementary functions except in very particular cases. However, since $\log f\left(x_{1}, \ldots, x_{j}\right)-\log f\left(k x_{1}, \ldots, k x_{j}\right)$ does not involve the integrals $I$, asymptotic formulae for the number of partitions of a multipartite number in which each part cannot occur more than a fixed number of times can be expressed entirely in terms of elementary functions.

Our references to the integral $I$ and its properties have been somewhat abbreviated in this note. However, in an article in preparation, one of the authors will discuss in detail the properties of both the integral $I$ and its generalization $I_{s r}^{\prime}$. The number $K\left(n_{1}, \ldots, n_{j}: A_{1}, \ldots, A_{j}\right)$ also appears to be of independent interest and it is our intention to investigate this in the near future.

\section{REFERENCES}

1. E. Grosswald, Some theorems concerning partitions, Trans. Amer. Math. Soc. 89 (1958), 113-128. MR 20 \#3840.

2. P. Hagis, $A$ problem on partitions with a prime modulus $p>3$, Trans. Amer. Math. Soc. 102 (1962), 30-62. MR 26 \#3688.

3. S. Iseki, A partition function with some congruence condition, Amer. J. Math. 81 (1959), 939-961. MR 21 \#7189. 1928.

4. K. Knopp, Theory and application of infinite series, Blackie, London and Glasgow,

5. J. Lehner, $A$ partition function connected with the modulus five, Duke Math. J. 8 (1941), 631-655. MR 3, 166.

6. J. Livingood, A partition function with the prime modulus $p>3$, Amer. J. Math. 67 (1945), 194-208. MR 6, 259.

7. I. Niven, On a certain partition function, Amer. J. Math. 62 (1940), 353-364. MR 1, 201.

8. H. A. Passi, An asymptotic formula in partition theory, Duke Math. J. 38 (1971), 327-337. MR 44 \#2722.

9. H. Rademacher, On the partition function $p(n)$, Proc. London Math. Soc. (2) 43 (1937), 241-254.

10. M. M. Robertson, Partitions of large multipartites, Amer. J. Math. 84 (1962), 16-34. MR 25 \#3919. 
11. M. M. Robertson, The evaluation of a definite integral which occurs in asymptotic partition theory, Proc. Roy. Soc. Edinburgh Sect. A 65 (1960/61), 283-309. MR 25 \#5046.

12. E. C. Titchmarsh, The theory of the Riemann zeta-function, Clarendon Press, Oxford, 1951. MR 13, 741.

13. E. M. Wright, Partitions of large bipartites, Amer. J. Math. 80 (1958), 643-658. MR 20 \#3111.

14. - The asymptotic behaviour of the generating functions of partitions of multi-partites, Quart. J. Math. Oxford Ser. (2) 10 (1959), 60-69. MR 21 \#3386.

DEPARTMENT OF MATHEMATICS, UNIVERSITY OF SURREY, GUILDFORD, SURREY, ENGLAND 